\title{
FORUM
}

\section{Europäische Paradoxien - ein Kommentar zur Lage der EU}

\author{
Sylvie Goulard*
}

Die Europäische Gemeinschaft ist fünfzig Jahre alt - ein Alter der Reife aber auch der Zweifel. Seltsamerweise reichen weder die Erfolge noch die vergangene Zeit aus, um das europäische Projekt in den Herzen und Köpfen der Menschen zu verankern. Fünfzig Jahre nach Unterzeichnung der Römischen Verträge wird der revolutionäre Charakter des gemeinschaftlichen Vorhabens immer noch nicht richtig wahrgenommen.

Der Europäische Rat vom Juni 2007 war Schauplatz bestürzender Gefechte: Die Forderungen der Staaten, die nicht einmal versucht haben den von ihnen unterzeichneten Verfassungstext ratifizieren zu lassen, waren regelrecht verblüffend. Die beeindruckende Leistung der deutschen Ratspräsidentschaft wurde im Negativen nur noch durch die Unaufrichtigkeit derjenigen Partner überboten, die bis zum Ende den Vorwand des niederländischen und französischen „Nein“ nutzten, um ihre Forderungen ständig zu verschärfen. Der Gegenwind war deutlich. Deshalb muss das unverhoffte Mandat für einen Reformvertrag verteidigt werden, so wenig mitreißend es auch sein mag.

Natürlich kann man den Eindruck der Krise relativieren. Ein Blick auf die Weltlage genügt, um zu zeigen wie marginal die Punkte sind, auf die sich die Meinungsverschiedenheiten der 27 Staats- und Regierungschefs konzentrierten und die sie drei Tage lang beschäftigten. In Europa werden die Menschenrechte umfassend respektiert, es herrscht Frieden, die Frauen sind frei wie nirgendwo sonst, kranke Menschen haben Zugang zu Pflegeeinrichtungen, ältere Menschen haben wenigstens das notwendige Minimum zum Leben. So können es sich die Europäer offenbar leisten, sich endlos über Kompetenzen oder Stimmengewichtung zu streiten und sind so sehr mit sich selber beschäftigt, dass sie jedes Maß und Ziel verlieren: wie unbekümmert muss man sein, den europäischen Lebensstil als gesichert zu betrachten! Und wie kurzsichtig, um zu glauben, dass man dieses Gut ewig bewahren könne ohne etwas für seinen Erhalt tun zu müssen.

Auch nach fünfzig Jahren ist die Europäische Union immer noch in drei Paradoxien verwickelt: im Hinblick auf ihre Institutionen, ihre Zielsetzung und ihre Grenzen.

\section{Die Paradoxie der Institutionen}

Die Notwendigkeit, sich gemeinsam einer Reihe neuer Herausforderungen zu stellen, steht außer Frage. Globale Probleme wie Fragen der Migration, des Terrorismus oder des Umweltschutzes sind akut. Hierbei sind die Nationalstaaten machtlos, solange sie einzeln agieren. ,Europa' allein ist nicht unbedingt die Lösung, denn auch unser Kontinent hat nicht die notwendige Größe um das Waldsterben, die Gletscherschmelze oder den mörderischen Wahnsinn Al-Qaidas zu bekämpfen. Das Argument, ,,in der Einheit steckt die Kraft“ ist un-

\footnotetext{
Dr. Sylvie Goulard, Präsidentin der Europäischen Bewegung Frankreich, Assoziierte Wissenschaftlerin am Centre d'Études et de Recherches Internationales, Paris.

Der Text basiert auf einem Vortrag, den die Verfasserin auf der wissenschaftlichen Konferenz ,50 Jahre Römische Verträge“" gehalten hat. Die Konferenz wurde vom Institut für Europäische Politik in Zusammenarbeit mit dem Arbeitskreis Europäische Integration und dem Centre International de Formation Européene in Berlin durchgeführt. Der Vortragsstil wurde weitgehend beibehalten.
} 
zureichend, um ,Europa ' in allen Fällen zu legitimieren. Allerdings stellt die Europäische Union heutzutage unbestreitbar den einzigen, von Erfolg gekrönten Versuch einer supranationalen Regierungsweise dar. Auch wenn sie alles andere als perfekt ist, so ist die Europäische Union doch demokratischer und misst der Rechtsverbindlichkeit eine größere Rolle zu, als jeder andere internationale Zusammenschluss. Gegründet wie die UNO, der Europarat oder Bretton Woods in den ersten Nachkriegsjahren, ist ihr Multilateralismus der einzige, der sich bewährt hat. Sie ist ein Prototyp, mit der Unvollkommenheit und dem unausgeschöpften Potenzial, das Prototypen kennzeichnet.

Die gewachsene Zahl an Mitgliedstaaten, die sich zwischen 1995 und 2007 von zwölf auf siebenundzwanzig vermehrt hat, ist eine weitere Herausforderung und Grund, die europäische ,governance‘ zu reformieren. Diese historisch gerechtfertigte, glückliche Erweiterung ist nicht ohne Folgen für das sensible Gleichgewicht der Institutionen. Dies zu ignorieren liefe darauf hinaus, den Kopf in den Sand zu stecken. Die Qualitäten der Neuen stehen außer Frage, doch ihre Zahl und ihre Heterogenität könnten als Zentrifugalkräfte wirken. Zwar hat das Parlament die Erweiterungen gut überstanden, doch der Ministerrat ist nur noch ein Schatten seiner selbst, bedenkt man beispielsweise seine Rolle in der Zeit als der Binnenmarkt ins Leben gerufen wurde (1986/1992). So berichten erfahrene ,Brüsseler', dass im Ausschuss der Ständigen Vertreter, die Diskussionen viel weniger intensiv als zuvor seien. Seitdem die Kommission zu einem Organ geworden ist, das entgegen seiner eigentlichen supranationalen Natur nunmehr aus einem Kommissar pro Land besteht, hat es viel an Glaubwürdigkeit verloren.

Ferner bildet das Streben nach mehr Demokratie eine weitere permanente Herausforderung für die Europäische Union. Man könnte viel über das angebliche Demokratiedefizit der Europäischen Union sagen, denn es ist im Wesentlichen auf die Zaghaftigkeit der Regierungen zurückzuführen, die zugleich den unvollendeten Zustand der Europäischen Union beklagen. Jedoch ist der Ruf nach mehr Demokratie ernst zu nehmen. In dem Stadium der Integration, in dem wir uns heute befinden, sind manche Mehrdeutigkeiten und Bastelarbeiten, die in den Anfangszeiten der europäischen Integration noch denkbar waren, unmöglich geworden. Die Zustimmung der Bevölkerungen zu gewinnen, ist heute eine unumgehbare Voraussetzung für einen dauerhaften Erfolg. Da unsere Gesellschaften sich von autoritären Modellen und Praktiken hin zu mehr konsensorientierten Formen weiterentwickelt haben, die versuchen viele unterschiedliche soziale Gruppen einzubeziehen und zu beteiligen, erwarten die Bürgerinnen und Bürger, dass die Entscheidungsprozesse flexibler und transparenter werden und Netzwerke allmählich an die Stelle hierarchischer Entscheidungssysteme treten. In Ländern wie Frankreich, wo die Lösung der Probleme stets Chefsache des starken Mannes an der Spitze des Staates bleiben soll, sind derartige Reformen ein harter Brocken!

Aus all diesen Gründen konnte die europäische Verfassung - die zwar nützliche Fortschritte beinhaltete, keinesfalls aber eine Neudefinition des europäischen Integrationsmodells (insbesondere nicht des institutionellen Dreiecks aus Kommission, Rat und Parlament) war, noch den Entscheidungsmechanismus in Frage stellte - nicht als die Lösung schlechthin verstanden werden.

Mit dem Verfassungsvertrag ist die Europäische Union zwar mit kleinen Schritten in die richtige Richtung gegangen, aber - und das trifft auch auf den Reformvertrag zu - sie ist auf halbem Wege stehen geblieben.

Die Methode mit der die Verfassung erarbeitet wurde, nämlich durch einen Konvent, der aus nationalen Abgeordneten bestand, die mit europäischen Abgeordneten, Regierungsvertretern und der Kommission in gutem Einvernehmen zusammen gearbeitet haben, war in sich selbst eine bemerkenswerte Innovation. Diese gegenseitige Entdeckung von ,Brüssel 
und dem politischen Personal der Mitgliedstaaten wurde durch die Tugend einer bis dahin unbekannten Transparenz in der EU-Diplomatie ergänzt. So waren alle Sitzungen und Dokumente des Konvents öffentlich. Die Zivilgesellschaft wurde auf bislang einmalige, systematische Weise befragt. Bedauerlicherweise sind es gerade diese Fortschritte, die mit dem französischen und niederländischen „Nein“ zunichte gemacht wurden: der Ball liegt nun wieder bei den Regierungen.

Auf das negative Votum, das den Bedarf an mehr Demokratie zum Ausdruck brachte, reagierten die EU-Regierungen wiederum mit Diplomatie unter Ausschluss der Öffentlichkeit. Wahrscheinlich hatte die deutsche Ratspräsidentschaft keine andere Wahl.

Mit Blick auf die erste Hälfte des Jahres 2007 wird klar, dass die großen Gewinner der durch die innere Rebellion in zwei Gründerstaaten ausgelöste Krise im Verfassungsprozess weder die Zivilgesellschaften, noch die Demokratie oder die öffentliche Debatte sind. Es sind stattdessen die Regierungen derjenigen Länder, die minimale Veränderungen wollen. Während zwei Länder den Verfassungsvertrag abgelehnt haben (Frankreich und die Niederlande) und 18 ihn ratifiziert haben (darunter mit Luxemburg und Spanien zwei durch ein Referendum), sind es gerade die Länder, die den Vertrag zwar unterzeichnet hatten, aber nichts taten, um ihr Versprechen zu halten, die die Anderen in Atem hielten und die die Forderungen in die Höhe trieben. Man kann in aller Sachlichkeit feststellen, dass die Anhänger des „Nein“, insbesondere die französische Linke, die sich als pro-europäisch und anti-liberal ausgab, Wasser auf die Mühlen derjenigen leitete, die sie angeblich bekämpfte.

Der Kompromiss vom Juni 2007 war nur möglich um den Preis, die Symbole wie die Flagge und Hymne zu opfern, die im Verfassungstext vorgesehen waren. Für die Pro-Europäer und die jungen Menschen deren EU-Zugehörigkeitsgefühl geweckt werden sollte, ist dies kein gutes Signal, auch wenn rechtlich gesehen der Status quo erhalten bleibt. Die Flaggen werden weiterhin auf den Dächern wehen. Der neue französische Europaminister JeanPierre Jouyet hat sogar die Heldentat vollbracht, die blaue Europaflagge mit den goldenen Sternen im ,salon de l'horloge“ zu hissen, und dies siebenundfünfzig Jahre nach der Schuman-Erklärung. Andere explizite Formulierungen, wie zum Beispiel der Vorrang des Gemeinschaftsrechts, verschwinden, ohne dass sich de jure etwas ändert. Diese Lösung ist zwar allemal besser als gar kein Vertragstext, sie ist aber nicht sehr ruhmreich.

Dagegen sind fast alle neuen Bestimmungen der Verfassung erhalten. Diese wurden übrigens nur wenig diskutiert und noch weniger in Frage gestellt - eine weitere Paradoxie, mit Ausnahme von zwei Fällen, auf die einzugehen sein wird.

Das Manöver der polnischen Regierung, die sich bereit erklärte „für Nizza zu sterben“ und dann jede sich bietende Möglichkeit der Mathematik nutzte, um sich zu profilieren (so eine Stimmengewichtung, die auf der Quadrat-Wurzel aus der Bevölkerungszahl beruht), soll hier unbeachtet bleiben. Um ihr Gesicht zu wahren erreichte die polnische Regierung, dass das Prinzip der doppelten Mehrheit erst in einigen Jahren angewendet werden wird. Auch wenn die Frist dafür verschoben wurde, ist das Prinzip der doppelten Mehrheit nun bemerkenswerterweise dennoch anerkannt. Auch die polnischen Autoritäten werden dann einsehen müssen, dass Größe stärker davon abhängt, Kompromisse zu schließen und damit Einigungen zu ermöglichen als davon, um jeden Preis das eigene Prestige zu verteidigen.

Besorgniserregender sind demgegenüber vor allem die Zugeständnisse an Frankreich und das Vereinigte Königreich. Der neue französische Staatschef hat das bekommen, was er erreichen wollte: Der Teil des Satzes von Artikel I-3 Abs. 2 VVE, in dem festgelegt ist, dass die Europäische Union ihren Bürgern ,einen Binnenmarkt mit freiem und unverfälschtem Wettbewerb" bietet, wurde gestrichen. Diese Bestimmung war 2005 einer der hauptsächlichen Streitpunkte innerhalb der gaullistischen Partei, weshalb Sarkozy durchaus geschickt 
agierte. Es wäre es jedoch passender gewesen, mit Geduld die Vorteile dieser Politik zu erklären, anstatt mit den Wölfen zu heulen.

Denn die gemeinschaftliche Wettbewerbspolitik ist sowohl ein soziales Instrument (wenn sie die Verbraucher und die kleinen Unternehmen gegenüber Kartellen schützt), aber auch ein Werkzeug der Macht, (das die Franzosen unterstützen sollten, da es sogar gegenüber den USA etwa bei der Fusionskontrolle oder im Falle von Microsoft Wirkung entfaltet) und eine Garantie gegen die Verschwendung öffentlicher Gelder (Verbot staatlicher Beihilfen). Gefährlich wäre ein Markt ohne freien und unverfälschten Wettbewerb, nicht das Gegenteil. In diesem Sinn sollte die Wettbewerbspolitik auch weiterhin betrieben, gar verstärkt werden, auch wenn sie natürlich nicht mit der Finalität der Europäischen Union gleichzusetzen ist. ${ }^{1}$ Jacques Delors hat sie immer in einem Dreieck gesehen, eingerahmt von Solidarität und Zusammenarbeit: ,die Zusammenarbeit befestigt, der Wettbewerb stimuliert, die Solidarität vereint".

Das andere besorgniserregende Zugeständnis an die Verfassungsgegner, ist das Opt-out des Vereinigten Königreichs von der Grundrechtecharta. Einem Mitgliedstaat zu ermöglichen, sich in einem so grundlegenden Punkt der Gemeinsamkeit in der Union von den Übrigen abzugrenzen, ist höchst zweifelhaft. Der Europäischen Union anzugehören und sich die Pflichten ,à la carte" auszuwählen, kann in Fragen technischer Angelegenheiten noch toleriert werden, es ist jedoch unerträglich in Fragen der Werte und Prinzipien. Es besteht damit das Risiko, den Zusammenhalt zu gefährden und, was London vielleicht übersehen hat, zukünftige Erweiterungen unwahrscheinlicher zu machen. Wie kann man von Beitrittskandidaten erwarten, dass sie die Werte der Union respektieren, wenn selbst manche EU-Mitglieder sich scheinbar demonstrativ davon distanzieren? Aus welchem Grund sollten sie ihre eigenen Traditionen, Sitten und Kultur aufgeben für EU-Europäer, die sich selbst in wesentlichen Fragen uneins sind? ${ }^{2}$

Zum heutigen Zeitpunkt weiß niemand so recht, wie die Zukunft des Reformvertrags aussehen wird: Entpuppt er sich als notwendiger, doch unzureichender Schritt, auf den bald der nächste größere folgen wird, um die neuen Aufgaben zu meistern? Auch wenn dies zur Scheidung von denjenigen führt, die nicht bereit sind so weit voranzugehen. Oder wird der Reformvertrag in einen Dauerzustand übergehen?

Um den Juni-Kompromiss zu erreichen, hat die deutsche Ratspräsidentschaft logischerweise diese Herausforderung als eine notwendige Vollendung des mit dem Vertrag von Maastricht 1992 begonnenen Zyklus bezeichnet. Keine „left overs“, forderte das Bundeskanzleramt. Das präzise Mandat, an dem der Bundeskanzlerin besonders gelegen war, verhinderte, das ,Sorgenkind“ einfach an die portugiesische Ratspräsidentschaft weiterzureichen. Sie hat sich der Verantwortung gestellt und damit allen Veto-Freunden und Muskelprotzen eine Lektion erteilt. Doch niemand sollte sich täuschen. Die wesentlichen Fragen bleiben offen. Dies ist die zweite Paradoxie und betrifft die Zielsetzung der europäischen Integration.

\section{Die Paradoxie der Zielsetzung: Was nützt uns die Europäische Union?}

Nach 50 Jahren europäischer Integration, nachdem Frieden erreicht und der Kalte Krieg überwunden worden ist, sind die Europäer nun von einer Art Erschütterung und tiefem Zweifel ergriffen.

Siehe Mario Monti: Nicolas Sarkozy, l'Europe et la concurrence, in: Le Figaro, 30.07.2007.

2 Sylvie Goulard: Britain's opt-out sets a dangerous precedent, in: Financial Times, 20.07.2007. 
Obwohl die Globalisierung die Fortsetzung des europäischen, multilateralen Experiments rechtfertigt, verlieren sich die Europäer in der Verlockung eines nationalen Rückzugs, des regionalen Partikularismus und den engen Horizonten des Lokalpatriotismus. Dies fällt besonders auf, wenn wir diesen kleinkarierten Zeitgeist mit dem der fünfziger Jahre vergleichen, als die Gründungsväter ,global“ dachten. ${ }^{3}$

Zwei Kernfragen interessieren uns besonders im Hinblick auf die Zielsetzung der europäischen Integration:

Die erste befasst sich mit der Rolle der Europäischen Union im Kontext der Globalisierung. Nützt Europa eigentlich noch etwas in dieser wirren, aber immer engeren Welt? Für Gordon Brown lautet die Antwort offenbar „nein“. Die Europäische Union habe ihre Daseinsberechtigung weitgehend verloren; im Kontext des Kalten Krieges geschaffen um den Frieden zwischen den Europäern zu verwirklichen, habe sie ihr Ziel erreicht. Auch wenn diese These nur wenig mit den wahren Motiven der Gründungsväter zu tun hat, scheint sie plausibel, blickt man etwa auf die unglaubliche Erfolgsgeschichte der Londoner City, die anstatt auf die kontinentale EU-Integration auf mehr Globalität gesetzt hat.

Wir sollten jedoch nicht vergessen, dass die anderen Staaten sich in einer anderen Lage als Großbritannien befinden. Manche haben ihre industrielle Basis erhalten oder ihre landwirtschaftliche Struktur nicht aufgegeben und besitzen zudem nicht den britischen Vorteil, dass ihre Muttersprache zugleich Lingua franca ist. Letztendlich wird uns die Geschichte lehren, ob die Briten die richtige Entscheidung getroffen haben, als sie strategische Sektoren, wie die Nahrungsindustrie und die heimische Produktion industrieller Güter (Medikamente, elektronische Geräte), aufgaben.

Auf der anderen Seite hat Nicolas Sarkozy bereits am Abend seiner Wahl zum Präsidenten der Europäischen Union vorgeworfen, ein ,trojanisches Pferd der Globalisierung“ zu sein und sie aufgefordert, uns besser vor den Folgen der Globalisierung zu ,schützen“. Folglich kommen in seinen Reden oft die Begriffe „gemeinschaftliche Präferenz“ und „Gegenseitigkeit" vor.

Die Mehrheit der europäischen Partner befindet sich zwischen diesen beiden Extremen. Sie wollen weiterhin eine starke Europäische Union aufbauen, ohne sich dabei zwangsläufig vor der externen Welt zu verschließen. Nicht ohne Spott machen sie zudem darauf aufmerksam, dass die Franzosen, verliebt in patriotische Töne, eine sehr offene Wirtschaft haben und starke global agierende Unternehmen besitzen, die im weltweiten Wettbewerb eine gute Figur abgeben.

Die Frage, die sich uns stellt ist abstrakt gesprochen nicht die des Nutzens der Europäischen Union. Abgesehen von einigen hartnäckigen Euro-Skeptikern, die aus der Europäischen Union austreten wollen, wünscht sich die britische Regierung eigentlich eine Stärkung des Binnenmarktes und sogar seinen Ausbau durch zukünftige Erweiterungen. Die Schlüsselfrage ist daher vielmehr, wie wir ein Gleichgewicht zwischen der legitimen Verteidigung eigener Interessen und heimischer Arbeitsplätze, der europäischen Wettbewerbsfähigkeit sowie dem notwendigen Fortbestand einer offenen Wirtschaft mittels kollektiver Entscheidungen erreichen können.

Da die Europäische Union auf dem Prinzip des freien Handels und der Überzeugung beruht, dass der freie Austausch Frieden und Wohlstand bringt, würde sie sich selbst verleugnen, wenn sie ,Öffnung ' plötzlich als schädlich ansehen würde. Sie darf sich aber auch nicht in der naiven Verteidigung eines puren und perfekten Marktes verirren, den es als solches

3 Sylvie Goulard: Le coq et la perle, 50 ans d'Europe, Paris 2007. 
nicht gibt. Sie kann es sich nicht erlauben, die verschiedenen Formen des Protektionismus zu ignorieren, die von anderen angewendet werden, seien sie offensichtlich oder versteckt.

Falls Gordon Brown und Nicolas Sarkozy in den kommenden Monaten ihre Meinungsverschiedenheiten öffentlich austragen sollten, dann würde die Europäische Union eine lebhafte und frontale, aber heilsame Auseinandersetzung erleben. Zu lange sind die Diskrepanzen ignoriert worden, sie müssen aber auf die Tagesordnung. Viele Europäer machen sich keine Illusionen darüber, dass der Globalisierungsprozess unaufhaltsam ist. Doch was sie noch weniger ertragen können ist, dass die entscheidenden Herausforderungen, die sich uns heutzutage stellen, totgeschwiegen werden.

Die Notwendigkeit, die Europäische Union im globalen Wettlauf zu positionieren, führt uns gleichzeitig dazu, die Frage nach der politischen Finalität des europäischen Projekts zu stellen. Und so sollte auch die richtige Reihenfolge aussehen um dies zu erreichen: nicht als eine Art Expertengespräch, sondern als Ergebnis tiefgreifender Reflexionen über die Zwänge und Handlungsmöglichkeiten in der realen Welt. Soll sich die Europäische Union zu einem Staat weiterentwickeln oder soll sie eine Organisation sui generis bleiben?

Wahrscheinlich ist dies der Gipfel der europäischen Paradoxie. Die meisten der Verantwortlichen und Beobachter lehnen jede Idee eines ,Superstaates' vehement ab. Auch wird dieser Begriff in vielen Reden als Schreckgespenst genutzt. Die Europäische Union solle etwas , anderes' bleiben. Gewiss, aber warum messen dieselben Personen die Europäische Union dennoch immerzu an einem Staat statt die sui generis Eigentümlichkeit zu erklären?

Um die Europäische Union, ihre Demokratie, Handlungs- und Leistungsfähigkeit zu beurteilen werden systematisch die Kriterien benutzt, die aus der Erfahrung mit den Nationalstaaten und deren Analyse resultieren. So ist das institutionelle Dreieck oft als undemokratisch beschrieben worden, da es nach dem Schema der Gewaltenteilung von Locke und Montesquieu beurteilt wird. Und doch wollte keiner der Europäischen Union jemals eine ,Regierung ' geben, die sich gegenüber einem ,Parlament` verantworten sollte. Die Exekutive besteht aus der Kommission, die vorschlägt, und dem Rat, der entscheidet. Die Legislative ist ebenfalls doppelköpfig; sie besteht aus dem Rat, als Kammer der Staaten, und dem Parlament, als Kammer der Völker. In Frankreich ist das beste Beispiel dieser Widersprüchlichkeit die permanente Forderung nach einer europäischen „Wirtschaftsregierung“ (gouvernement économique) und die gleichzeitige vehemente Ablehnung des „Föderalismus“. So etwas wie eine „Wirtschaftsregierung“ gab es freilich noch nie: Tatsächlich existieren nur Regierungen, die mit gesamtgesellschaftlichen Aufgaben und den Feldern der Wirtschaft, Umwelt, des Sozialen, der Justiz und so weiter betraut sind. Ein einzelnes Feld herauslösen zu wollen, ohne dabei die gesamte Konstruktion neu zu definieren und die entsprechenden Kompetenzen zuzuweisen, erscheint fragwürdig.

Auch die Vergleiche mit den USA sind ungezählt: wie viel reaktiver ihr Federal Reserve System (Zentralbank-System) sei, wie viel fähiger dort Politik gemacht würde, wie viel besorgter die Hüter des Wettbewerbs dort seien und sich für die amerikanischen Interessen einsetzten, und Vieles mehr. Aber sind die USA nicht leistungsstärker, weil sie - mit ihrer föderalen Struktur - besser organisiert sind, um ihre eigenen Interessen besser zu verteidigen? Wer hindert die Europäer daran, dasselbe zu tun? Und wer wird diejenigen zum Schweigen bringen, die sich immer jammernd über unsere Mängel beschweren und jeglichen Fortschritt ablehnen? Denn wir haben das Europa, das wir verdienen.

Es ist umso bedauerlicher, da die Vielfalt der föderalen Modelle eigentlich beruhigend wirken müsste. Um den Vergleich mit den USA weiterzuführen: Es ist zu beobachten, dass die Amerikaner über eine Vielfalt an Steuer-, Wirtschafts- und sogar Strafrechtssystemen verfügen. Nicht zu vergessen, dass sogar eine so grundlegende Frage wie die Anwendung 
der Todesstrafe den Bundesstaaten überlassen wird! Die Europäische Union betreibt Föderalismus wie Molières Monsieur Jourdain Prosa: Ihr oberster Gerichtshof, ihre Zentralbank, ihre Kommission, soweit sie über den Wettbewerb entscheidet, sie alle sind föderale Organe. Damit leben sogar die Engländer. Kein Grund also, daraus ein Drama zu machen.

Früher oder später werden wir uns mit diesen Fragen der Finalität auseinandersetzen müssen, denn das Mandat für die Regierungskonferenz 2007 bleibt hinter dem Laekener Mandat vom Dezember 2001 und dem Europäischen Konvent zurück. Nach dem Schock der gescheiterten Referenden leisteten die Regierungen damit nur einen minimalen Beitrag. Doch die Fragestellung wird sich immer wieder erneut stellen, denn man kann den Europäern nicht ewig erzählen „Du wirst es verstehen, wenn du größer bist““.

Schon während der Botschafterkonferenz Ende August 2007 hat Nicolas Sarkozy, eifriger Befürworter eines „Mini-Vertrags“, den Vorschlag gemacht, einen „Rat der Weisen“ einzurichten, der über das „,europäische Projekt“ nachdenken soll. „Zwanzig Mal sollst du dein Werk überarbeiten“, pflegte Boileau dazu im XVII. Jahrhundert zu sagen.

Unhabhängig von der Entscheidung des Europäischen Rates im kommenden Dezember, dem französischem Vorschlag Rechnung zu tragen oder nicht, muss die Frage nach der Zielsetzung der Europäischen Union ohnehin behandelt werden - und dies vor allem wegen der dritten Paradoxie, der äußersten Grenzen der Europäischen Union.

\section{Die Paradoxie des Territoriums: Bis wohin soll die Europäische Union reichen?}

Kanzler Kohl erzählte oft, dass er als Jugendlicher die Grenzpflöcke an der Grenze zwischen Frankreich und Deutschland wegtrat. Für die Generation der Baumeister der Europäischen Integration war die Abschaffung der Grenzzäune und Abschottungen eine der Daseinsberechtigungen Europas schlechthin. Doch in dem Stadium der Ausdehnung und Integration, in dem sich die Europäische Union heute befindet, kann sie es sich nicht erlauben, ihre überkommene Vorgehensweise nicht zu verändern. ${ }^{4}$ Sollte sie dies nicht tun, wird sie an den unüberwindbaren Ängsten zugrunde gehen, die sie ausgelöst hat. Schon die Sorge vor endlosen Erweiterungen hat in den beiden negativ ausgegangenen Referenden eine Rolle gespielt. In Frankreich, wo sich eine Mehrheit gegen den Beitritt der Türkei aussprach und in den Niederlanden, wo die Menschen mit einer Identitätskrise zu kämpfen haben. Der Gedanke an eine grenzenlose Ausdehnung zerstört jegliche Aussicht auf ein Zugehörigkeitsgefühl und schwächt zudem die Gemeinsame Außen- und Sicherheitspolitik (GASP). Wie kann man eine ,Außenpolitik' betreiben, ohne sich der Außenwelt bewusst zu sein?

Es sollte eine Lehre sein, dass die Öffentlichkeit gegenüber der letzten Erweiterungsrunde, so historisch gerechtfertigt sie auch war, sehr zurückhaltend war. Denn die Besorgnis der Bürger darüber, nicht zu wissen ,wie weit es denn weitergehen wird“ ist nicht zu ignorieren. Die Europäer stellen sich mit gesundem La Fontaine'schen Menschenverstand die Frage, ob die Europäische Union sich nicht für ,den Frosch hält, der so groß sein wollte wie der Ochse“. Wenn die Sorgen der Bürger vor Überdehnung und Überforderung der Europäischen Union nicht beantwortet werden, werden Xenophobe und andere Extremisten dies auszunutzen wissen.

Die mühsamen Versuche, eine institutionelle Reform durchzubringen, lassen Zweifel an der Vitalität der Europäischen Union aufkommen, auch die Knappheit der Budgetmittel ist den Bürgerinnen und Bürgern bekannt. Da sie von den Regierungen ständig hören, die Europäische Union solle sich den neuen Bedürfnissen der Bürger zuwenden, fordern sie folge-

4 Für eine umfassende Analyse siehe auch: Sylvie Goulard: Le Grand Turc et la République de Venise, Paris 2005; deutsche Version: EU-Türkei: eine Zwangsheirat?, Berlin 2006. 
richtig ein, dass sie mehr in die Auswahl der EU-Mitglieder involviert werden. Um kulturelle und andere Unterschiede und Gegensätze überwindbar zu machen bedarf es der Annäherungen, für die jedoch aktive, engagierte oder zumindest konsultierte und zustimmende Bürger vonnöten sind.

Eine endlose Erweiterungspolitik eröffnet unseren Nachbarn sicherlich Perspektiven - so wird die Erweiterungspolitik auch oft gerechtfertigt. Dabei könnte jedoch gerade diese Erweiterungspolitik für die Europäer auch Perspektiven schwinden lassen, denn während außerhalb der Europäischen Union Hoffnungen erweckt werden, wird innerhalb der Europäischen Union Verzweiflung genährt. Für einige, vor allem in Frankreich, aber auch in Österreich, würde die Fortsetzung der Erweiterung bedeuten, dass die Suche nach einer politischen Identität der Europäischen Union aufgegeben wurde, ebenso wie die gegenseitige finanzielle Unterstützung und die Hoffnung auf den Erhalt hoher sozialer Standards.

Einige möchten die Europäische Union auf Basis von Kriterien wie Geografie, Geschichte und Religion eingrenzen. Diese Kriterien, insbesondere das letzte, sind jedoch in einer weltoffenen und säkularen Union, in der es ohnehin schon eine Vielfalt an Religionen gibt, äußerst fragwürdig und nicht zuletzt unwirksam. Die Antwort steht in keinem Buch, sie ist stattdessen politischer Natur und setzt voraus, dass sich die Europäer über ihr gemeinsames Vorhaben, ihre Weltanschauung und ihr Schicksal äußern, ohne sich dabei vor großen Worten zu scheuen - ein wahrlich großes Vorhaben, das man bisher umgangen hat. Womöglich war es damals klüger, nicht zu genau auf diese unangenehmen Fragen einzugehen. Nun ist das ,Familiengeheimnis ‘ aber zur Last geworden, nun verlangen die Kinder Rechenschaft.

Es geht um folgende Schlüsselfragen:

- Wie viel Souveränitätsaufteilung akzeptieren wir? Dies brächte uns zur Frage der Finalität zurück.

- Wie viel Mittel sind wir bereit, dem Gemeinschaftsbudget im Namen der Solidarität zu übertragen?

- Welches Zugehörigkeitsgefühl können wir hervorrufen, damit eine ausreichende Akzeptanz der Bürgerinnen und Bürger erzielt werden kann?

Keine dieser Fragen ist einfach zu beantworten. Doch je mehr wir schweigen, desto mehr erhärten wir die europäischen Zweifel. Glücklicherweise ist ein Wandel gegenüber 2002/ 2004 spürbar. Die Kommission sowie der Rat haben eingesehen, dass der „Erhalt der Stoßkraft des europäischen Integrationsprozesses“" notwendig ist. Die Wiederbelebung dieses sogenannten vierten Kopenhagener Kriteriums, das lange vergessen worden war, scheint darauf hinzuweisen, dass die Europäische Union Erweiterungsfragen nun weniger politisch korrekt angeht und verstärkt die Anliegen der EU-Europäer sowie das Überleben der Europäischen Union als politisches Projekt berücksichtigt.

Auch sollten wir nicht vergessen, dass Artikel 49 des EU-Vertrags sich darauf beschränkt, den europäischen Nachbarn, die den Kriterien entsprechen, stets nur die Möglichkeit in Aussicht zu stellen, der Europäischen Union beitreten zu können, nicht jedoch das Recht auf Mitgliedschaft einräumt. Ihnen Zugang zu gewähren bedeutet für die Europäer eine Eventualität, die sie zur Kenntnis nehmen müssen, nicht jedoch eine Verpflichtung.

Die Wahl von Nicolas Sarkozy ist wahrscheinlich eine Wende in der Erweiterungspolitik. Denn zum ersten Mal sprach sich ein Amtsinhaber und Staatschef, Mitglied des Europäischen Rates, energisch gegen die Fortsetzung der EU-Erweiterung aus und verneinte zugleich, dass die Türkei zu Europa gehöre. Doch als Präsident der Republik ist er der staatlichen Kontinuität verpflichtet. Deswegen hat er Ende August auf der Botschafterkonferenz erklärt, dass er sich nicht gegen die Fortführung der Verhandlungen aussprechen werde. Al- 
lerdings nur bei jenen Kapiteln, die nicht das Ergebnis des Prozesses (Beitritt oder privilegierte Partnerschaft) vorentscheiden werden.

Eine Paradoxie in der Paradoxie ist, dass sein Vorgänger Jacques Chirac, persönlich ein Befürworter des türkischen Beitritts, den Prozess durch eine neue Bestimmung in der französischen Verfassung gefährdet hat, wonach nun vor jeder neuen Erweiterung ein Referendum in Frankreich durchgeführt werden muss. Wer kann garantieren, dass eine solche Volksabstimmung positiv ausgehen wird? Die Vergangenheit hat gezeigt, dass die Wähler oft eine andere Frage beantwortet haben, als jene, die gestellt worden war. Mit einem derartigen Thema, das demagogische und fremdenfeindliche Versuchungen birgt, hat Präsident Chirac ein erhebliches Risiko in Kauf genommen. Schon wegen dieses Damoklesschwerts sollten sich die Europäer eine alternative Lösung überlegen. Dramatisch wäre es, wenn die Bevölkerung eines Beitrittslandes, welches die Forderungen der Europäischen Union erfüllt hat, durch den Willen der Bevölkerung eines Mitgliedstaates ausgeschlossen würde. Um dieses Szenario zu verhindern, müssen alle Möglichkeiten diskutiert werden, von einer umfassenden Begleitung der Verhandlungen, mit einer Teilnahme der europäischen Zivilgesellschaft bis hin zum Verzicht auf den ursprünglichen Plan der Mitgliedschaft. Nichts sollte tabu sein. Denn die Verschiebung dieser Debatte, würde die Sache am Ende nur verschlimmern.

\section{Schlussbemerkung}

Am Ende dieser Reise in das Land der Paradoxien, ist nur eines sicher; dass eine starke europäische Gemeinschaft mit der Fähigkeit, sich den globalen Herausforderungen entgegenzustellen, eine Notwendigkeit bleibt.

Die Europäische Union ist als „Labor der Globalisierung“ (Delors) und als Prototyp der global governance unverzichtbar. Sie ist der einzige Versuch, der je unternommen wurde, eine politische Organisation zu schaffen, in der Gesetze, Solidarität und Demokratie auf supranationaler Ebene vorhanden sind. In einer Welt, in der viel geredet und wenig realisiert wird, bildet die Europäische Union eine Ausnahme. Sie ist gewiss nicht perfekt und bedarf der Verbesserung, sie benötigt ernsthafte Reformen und Infragestellungen. Aber mehr noch müssten die Europäer die Paradoxien wahrnehmen, die ihre Handlungsfähigkeit einschränken und ihre Erfolgschancen mindern.

Übersetzung aus dem Französischen von Martin Bafoil und Gesa-Stefanie Brincker. 